\title{
Application of infrared thermography for determining the temperature distribution in Taylor's impact test
}

\author{
by L. KRUSZKA $(*)$, W.K. NOWACKI $\left({ }^{* *}\right)$ and W. OLIFERUK $\left(^{* *}\right)$
}

\begin{abstract}
1*) Military Technical Academy, ul. Kaliskiego 2, 00-908 Warsaw, Poland.
$1^{* *}$ Institute of Fundamental Technological Research, Polish Academy of Sciences, ul. Swietokrzyska 21, 00-049 Warsaw, Poland.
\end{abstract}

\section{Abstract}

The object of the work is to measure the temperature field in solids (aluminium) during its dynamic plastic deformation at high strain rates. The present paper describes the testing stand based on Hopkinson's pressure bar system and AGA thermovision set, details of the experiment and analysis of the experimental results.

\section{Introduction}

The main aim of the present work was to determine the temperature distribution along a cylindrical aluminium specimen during its dynamic plastic deformation at strain rates of the order of $10^{2}-10^{3} \mathrm{~s}$. This range of strain rate was obtained in Taylor's impact test. The specimen struck normally an obstacle with the initial velocity $v_{0}$ undergoing considerable deformation. It was recognised that the most suitable measuring technique of temperature distribution in dynamically deformed specimens, is a no-contact measurement based on the infrared radiation (IR) detection.

\section{Experiment}

The testing stand was based on Hopkinson's split pressure bar system and AGA 680 thermovision set (TS). In this set indium antimonide cooled by liquid nitrogen was used as the IR-sensitive detector. The selected elements of this stand are shown in the figure 1. The aluminium specimen 1 moved inside the barrel 2 of a pneumatic launcher towards the obstacle 3 that was an incident bar of split Hopkinson pressure bar. The impact velocity $v_{0}$ was determined by measuring the time between cuttings of two parallel light beams by the impinging specimen. These velocities were about $75-150 \mathrm{~ms}^{-1}$.

In order to determine the temperature distribution in the most deformed part $B-C$ of the specimen, the impact space was observed by a thermovision camera 4 . The thermovision image is a scanning result of an observed surface by two germanium prisms rotating vertically and horizontally. This image on a screen of a display unit 5 corresponds to the IR power surface distribution. Duration of the deformation process was too short (about $2.10^{-4} \mathrm{~s}$ ) to observe the accompanying increase of temperature on a whole image $\left(6.10^{-2} \mathrm{~s}\right)$. Hence, in our experiment, the horizontal prism was stopped. It was then possible to obtain a frequency of image line equal to $1.6 \mathrm{kHz}$. The registering time of this line on the screen was $625 \mathrm{~ms}$. This line corresponds to a generating line of the cylindrical specimen. The investigated thermomechanical process was axially symmetric. Such an approach was sufficient to properly describe the above impact process. The amplitude of the thermovision signal is a function of temperature difference between an investigated specimen surface and a space nearby. The temperature of this space varied in an uncontrolled way and, thus, it was difficult to determine the absolute temperature of the specimen. To solve this problem, an indicator of constant 
temperature A was placed in the observation space of the camera. This indicator consisted of a resistance wire fed with a stabilised current. The temperature of this indicator does not need be known. This temperature played a role of constant reference level. An example of a typical IR power distribution along the specimen generating line, recorded by a digital storing oscilloscope 6 , is shown in the figure 2 . In this figure, $\xi$ is the IR power, $t$ the time and THV, the sensitivity of TS.

\section{Calibration of the temperature measuring set}

In order to find a relation between the specimen surface temperature and the emitted IR power (i.e., to determine its surface temperature) the specimen was replaced by a small aluminium tank. Inside this tank a water flowed from a temperature-regulating thermostat. The tank surface and the constant temperature indicator were in the observation space of the thermovision camera. The controllable way of the changing of the tank surface temperature (measured by a thermocouple) made possible the calibration of level differences on the display unit screen between the indicator and successive altitude points of the thermovision image.

\section{Results}

Using the calibration of the temperature measuring set it was possible to assign the absolute temperature values to the recorded IR power distribution emitted by the points of the specimen meridian line. The figure 3 illustrates a registration of the temperature distribution along the observed segment of the length $L_{k}=58.9 \mathrm{~mm}$. By changing the frequency of the oscilloscope time base, it was possible to record the sequential time intervals of $\Delta t_{k}=625 \mathrm{~ms}$ in duration. The maximum heating of the specimen surface occurred in the second observing sequence. It proves a thermal inertia of the specimen. In this figure $\Delta t$ denotes the time interval of a jump of the thermovision camera prism to the next observation sequence. This experiment allowed to determine the maximum temperature values $T$ in function of the impact velocities $v_{0}$ figure 4 , in the considered dynamic deformation process. In this figure, $T_{0}$ is the room temperature.

\section{Estimation of temperature measurement error}

The temperature measurement error in the present experiment depends on the following factors: a temperature stability of the resistance wire determining the constant reference level, a temperature resolution of TS, an accuracy of the tank surface temperature measurement and a reading off error. The temperature stability of the indicator was determined experimentally by a comparison with a temperature of a black body model. Its value was \pm $3 \mathrm{~K}$. The temperature inaccuracy of the used model, according to a catalogue data, is $\pm 0.5 \mathrm{~K}$. The error of the tank surface temperature measurement was equal to $\pm 1 \mathrm{~K}$. The temperature resolution of TS for the value about $343 \mathrm{~K}$ is $\pm 0.1 \mathrm{~K}$. The error of the electric signal measurement and reading off error were negligible. In the worst case, the mentioned factors might sum up. Therefore, the absolute temperature measurement error is below $\pm 4.6 \mathrm{~K}$. Its relative value is not larger than $10 \%$. This error can be considerably and easily decreased by improving the feeding stability of the resistance wire in the reference indicator. 


\section{Conclusions}

The determined temperature field was necessary to verify experimentally a theoretical description of the cylindrical specimen impinging on the obstacle [1]. The present experiment proved the usefulness of the infrared thermography for the investigation of phenomena at dynamic deformations of metals.

[1]. KRUSZKA (L.), NOWACKI (W.K.). - On the large strain coupled thermoplastic analysis in normal impacting long cylindrical specimen, Inelastic Solids and Structures, Antoni Sawczuk Memorial Volume, Pineridge Press, 1990, Swensea, U.K.

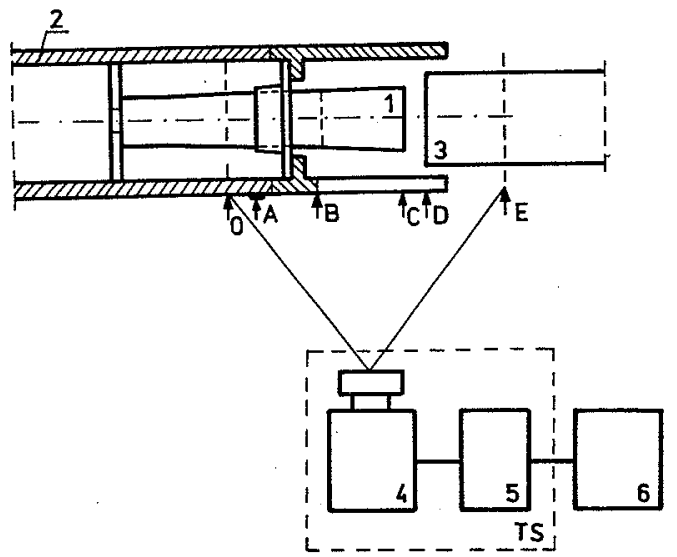

Fig. 1. - Selected elements of testing stand

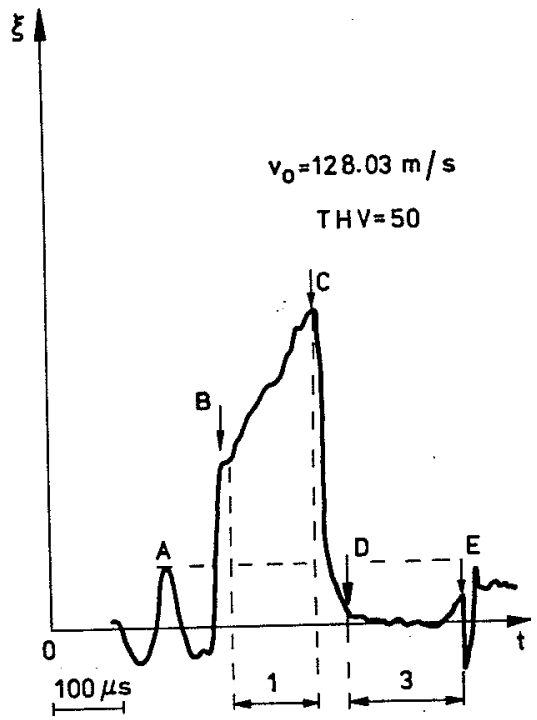

Fig. 2. - Typical infra-red radiation power distribution 
http://dx.doi.org/10.21611/qirt.1992.023

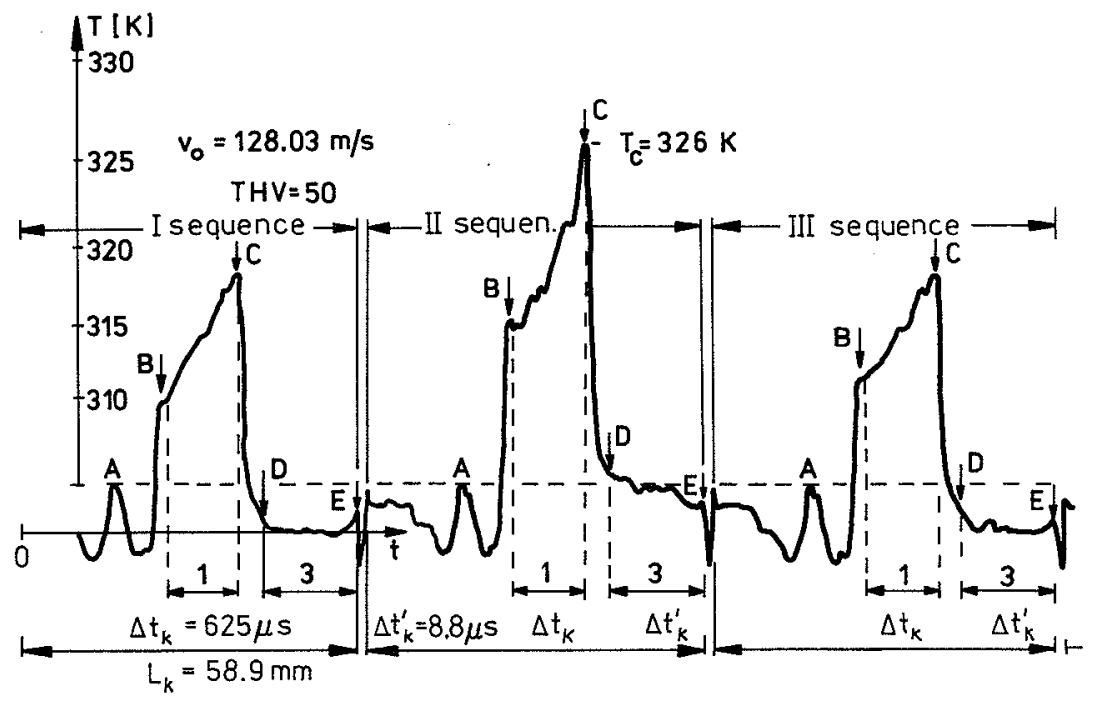

Fig. 3. - Temperature distribution along observed segments

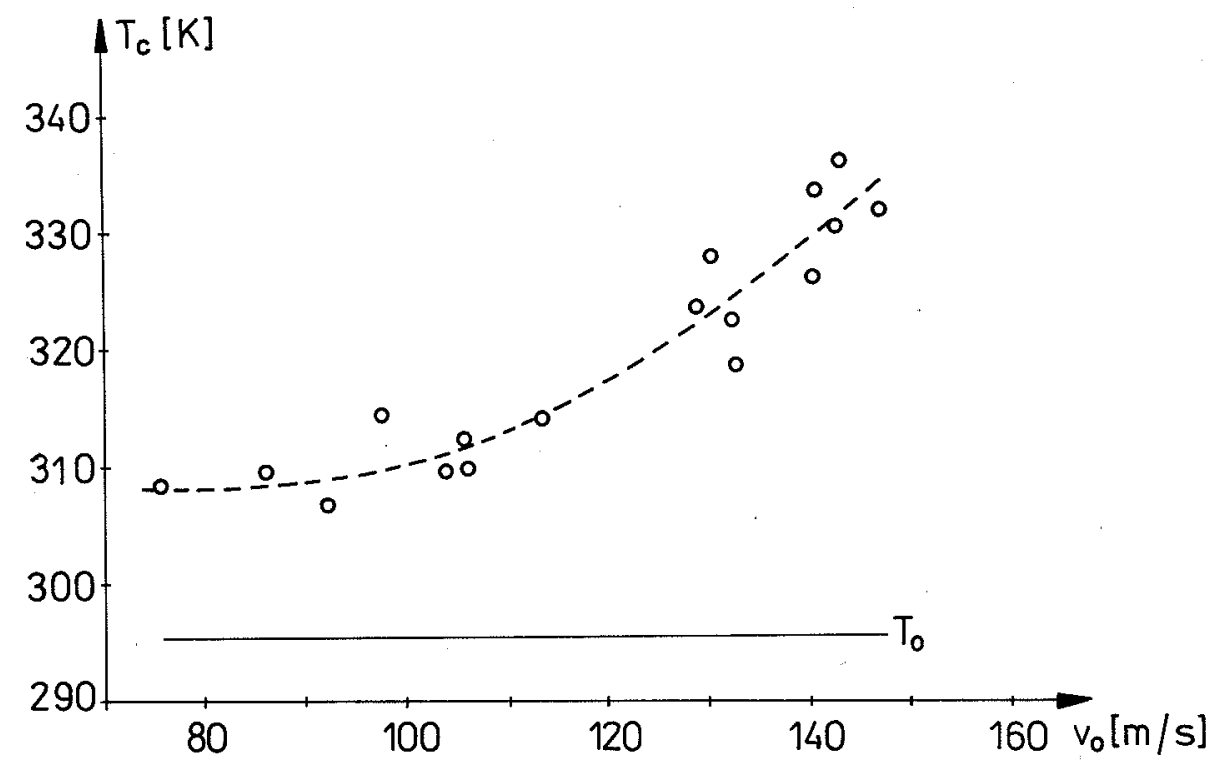

Fig. 4. - Temperature at impacting face of specimens versus impact velocity 